\title{
Analyses of zonal atmospheric excitation functions and their correlation with polar motion excitation functions
}

\author{
J. Nastula, W. Kosek, B. Kolaczek \\ Space Research Centre, Polish Academy of Sciences, Bartycka 18a, PL-00-716 Warsaw, Poland
}

Received: 22 January 1996 / Revised: 10 March 1997 / Accepted: 27 March 1997

\begin{abstract}
The atmospheric influence on the Earth's, rotation can be described by the effective atmospheric angular momentum (EAAM) functions. In this study we focus on the analysis of short period variations of the equatorial components of the zonal EAAM excitation functions $\chi_{1}$ and $\chi_{2}$ and their influence on similar variations of polar motion. The global objective analysis data of the Japanese Meteorological Agency for the period 1986-1992 were used to compute the EAAM excitation functions in different latitude belts. Time- and latitude-variable amplitude spectra of variations of these functions with periods shorter than 150 days, containing pressure, pressure with the inverted barometric correction, and wind terms were computed. The spectra show distinct latitude and time variations of the prograde and retrograde oscillations which reach their maxima mainly in mid-latitudes. Prograde and retrograde oscillations with periods of about 40-60 days and about 110-120 days are seen in the spectra of pressure terms of the equatorial components of the zonal EAAM excitation functions. Additionally, correlation coefficients and cross-spectra between variations of the geodetic polar motion and equatorial components of the zonal EAAM excitation functions were computed to identify the latitude belts of the globe over which atmospheric circulation changes are correlated mostly with short period variations of the polar motion excitation functions. The correlation coefficients vary in time and latitude and reach maximum values in the northern latitudes from $50^{\circ} \mathrm{N}$ to $60^{\circ} \mathrm{N}$. In the cross-spectra between the polar motion excitation functions and pressure terms of the zonal EAAM excitation functions there are peaks of common prograde oscillations with the periods around $20,30,40-50,60$ and 80-150 days and of common retrograde oscillations around 20,30, 40 and 50-70 days.
\end{abstract}

Correspondence to: J. Nastula nastula@cbk.waw.pl Paper presented at the IERS Workshop in Paris, March 1994

\section{Introduction}

Atmospheric influences on the Earth's rotation are described by the EAAM excitation functions (Barnes et al., 1983). These functions can be partitioned into contributions from zonal winds $(w)$ and surface pressure $(p)$. If we assumed that the ocean responds to the overlying atmospheric pressure completely isostatically, as an inverted barometer, the barometric correction $(i b)$ is invoked (Munk and McDonald, 1960). The atmospheric perturbations of the polar motion can be expressed by equatorial components of the EAAM excitation functions $\chi_{1}$ and $\chi_{2}$, the axes $\chi_{1}$ and $\chi_{2}$ are directed along the Greenwich Meridian and $90^{\circ} \mathrm{E}$. respectively (Barnes et al., 1983). The atmospheric contributions to the short period oscillation to the short period oscillations of the polar motion in time scales from several weeks to several months and amplitudes ranging from 2 to 10 mas have been shown by many authors (Barnes et al., 1983; Brzeziński, 1987; Chao, 1993; Eubanks et al., 1988; Hide and Dickey, 1991; Kosek et al., 1995; Kuehne et al., 1993; Nastula, 1992, 1995a; Nastula et al., 1990). Several authors demonstrated that these correlations vary with time (Kuehne et al., 1993; Nastula, 1992, 1995a; Kosek et al., 1995).

Until now the relationship between the variations of regional EAAM and polar motion excitation functions has not been known so well as the relationship between the variations of global or hemispheric EAAM excitation functions and the Earth's rotation, although the equatorial components $\chi_{1}$ and $\chi_{2}$ of some regional EAAM excitation functions were determined in the past (Salstein and Rosen, 1989; Nastula, 1995b). To derive such data access to a huge amount of grid point data is necessary. The analysis of the regional EAAM excitation functions has shown that their pressure components, computed over different Earth surface regions, can be useful in studies of regional contributions to global phenomena. For example, strong variations of 
surface pressure found over the North Atlantic and North Pacific, lead to the conclusion that these areas are important in studies of polar motion variations over intraseasonal time scales (Salstein and Rosen, 1989).

Time- and latitude-variable spectra of variations of the zonal EAAM excitation functions were computed in order to find the importance of different latitude belts in producing the EAAM excitation functions.

Our analysis of correlation coefficients differ from the previous investigations mainly in the use of zonal instead of global EAAM data. In addition, the analysis shows for the first time the latitude- and time-variable variations of amplitudes of zonal EAAM short period oscillations, separated into prograde and retrograde parts. For these computations the new Fourier transform band pass filter (FT BPF) spectrum method of complex-valued time series was applied (Kosek, 1995; Popiński and Kosek, 1995).

\section{Computation of the zonal EAAM excitation functions}

The global and hemispheric values of the EAAM excitation functions are routinely issued by the Japanese Meteorological Agency (JMA) at 12 or $6 \mathrm{~h}$ intervals (Naito et al., 1987; Salstein and Kann, 1993). In practice, three components of the EAAM excitation functions, two equatorial ones $\chi_{1}, \chi_{2}$, and one axial one $\chi_{3}$, are computed by different meteorological centers. For studies of latitude variations of the EAAM excitation functions we computed homogeneous sets of equatorial components of the zonal EAAM excitation functions for different latitude belts using the formula given by Barnes et al. (1983). For these computations the Global Objective Analysis Grid Point data (GANL), routinely determined and stored by the JMA (Naito et al., 1987), were used. The characteristics of the GANL data are shown in Table 1.

The zonal EAAM excitation functions $\chi_{1}$ and $\chi_{2}$ were computed in latitude belts of $22.5^{\circ}$ latitude width for the years 1986.7-1992.6 (Fig. 1) and in latitude belts of about $2^{\circ}$ latitude width, covering the whole globe for the years 1988.3-1992.6 for 00 and 12 UTC (Fig. 2). These functions contain the pressure $(p$ nib), pressure with inverted barometric correction ( $p i b)$, and wind $(w)$ terms. The new topographic model, based on the Global Digital Terrain Model (TUG87), was adopted (Sunkel and Wieser, 1987).

Some examples of variations of the computed equatorial components of the zonal EAAM (JMA) excitation functions are presented in Figs. 1 and 2. In the northern latitude belts a distinct annual oscillation in $\chi_{1}, \chi_{2}$ and especially in the pressure terms can be seen (Fig. 1). The

Table 1. Characteristics of GANL data from Japanese Meteorological Agency

\begin{tabular}{lll}
\hline Period & Pressure level & Mesh-degrees \\
\hline $1986.7-1988.3$ & 14 levels from 850 to $10 \mathrm{mb}$ & $25^{\circ} \cdot * 25^{\circ}$. \\
$1988.3-1992.6$ & 15 levels from 1000 to $10 \mathrm{mb}$ & $1875^{\circ} \cdot{ }^{*} 1875^{\circ}$. \\
\hline
\end{tabular}

amplitudes of the wind terms in each of the eight latitude belts of $22.5^{\circ}$ latitude width are several times greater than the amplitudes of the pressure terms. In the case of the whole hemispheres the situation is opposite: the amplitudes of the wind terms are several times smaller than the pressure term amplitudes (Nastula, 1995a). It is obvious that in the global EAAM excitation functions the zonal wind variations cancel each other while the pressure variations act in concert.

There is a characteristic large positive maximum in the pressure terms of $\chi_{2}$ in the Northern Hemisphere, either modified or not by inverted barometric correction, and a smaller negative minimum in the Southern Hemisphere (Fig. 2). The $\chi_{1}$ components have smaller maxima than $\chi_{2}$ and similar amplitudes in the Northern and Southern hemispheres. The wind terms of $\chi_{1}$ and $\chi_{2}$ reach similar maximum values in the northern and southern mid-latitudes.

\section{Latitude- and time-variable spectra of short period variations of the zonal EAAM and polar motion excitation functions}

In this section latitude and time variations of the zonal EAAM excitation functions of the complex-valued vector $\chi=\left(\chi_{1}+i \chi_{2}\right)$ are analysed.

To investigate short period oscillations of the zonal EAAM excitation functions the long period oscillations were removed using the Butterworth high pass filter (HPF) with a cutoff period of 150 days (Otnes and Enochson, 1972)

To compute the latitude- and time-variable spectra of short period $\chi$ oscillations the method of the FT BPF spectrum complex-valued time series was applied (Kosek, 1995; Popiński and Kosek, 1995).

\subsection{Latitude-variable spectra}

In this case the latitude-variable spectrum estimation is given by the following formula (Kosek, 1995; Popiński and Kosek, 1995):

$$
\hat{S}(\phi, T)=\frac{1}{N-2 N_{f}} \sum_{t=N_{f}+1}^{N-N_{f}}\left|u_{\phi, t}(T)\right|^{2},
$$

where $N$ is the number of points, $N_{f}=10$ is the number of points to be cut off at the beginning and end of time series due to filtration errors, $u_{\phi, t}(T)=F T^{-1}$ $\left[F T\left(\chi_{\phi, t} A(T, B)\right]\right.$ is a complex-valued oscillation with a period $T$ computed using the FT BPF from the zeromean $\chi_{\phi, t}$ time series, corresponding to a particular latitude zone $\phi$, and $A(T, B)$ is the parabolic transfer function in which the filter bandwidth $B=0.006$ was adopted (Popiński and Kosek 1995; Kosek 1995). The parameter $B$ defines the frequency resolution of a spectrum.

A single spectrum point is the variance estimate of a complex-valued oscillation computed using the FT BPF. 


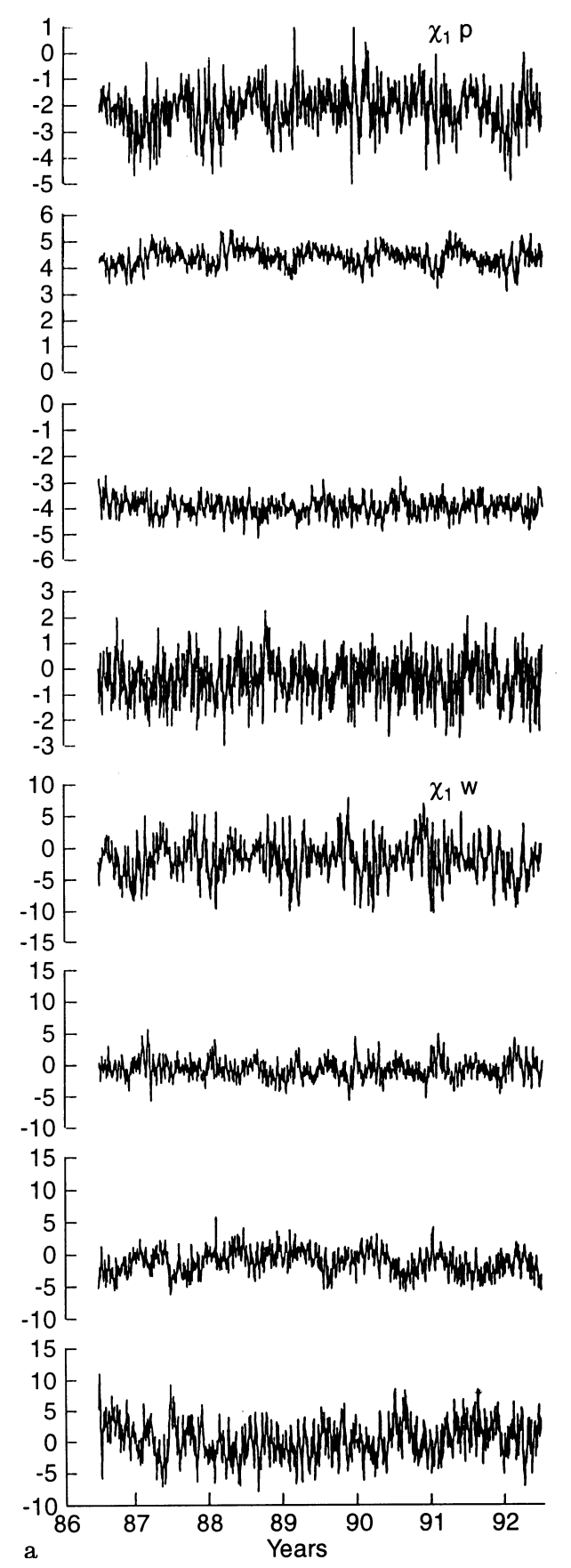

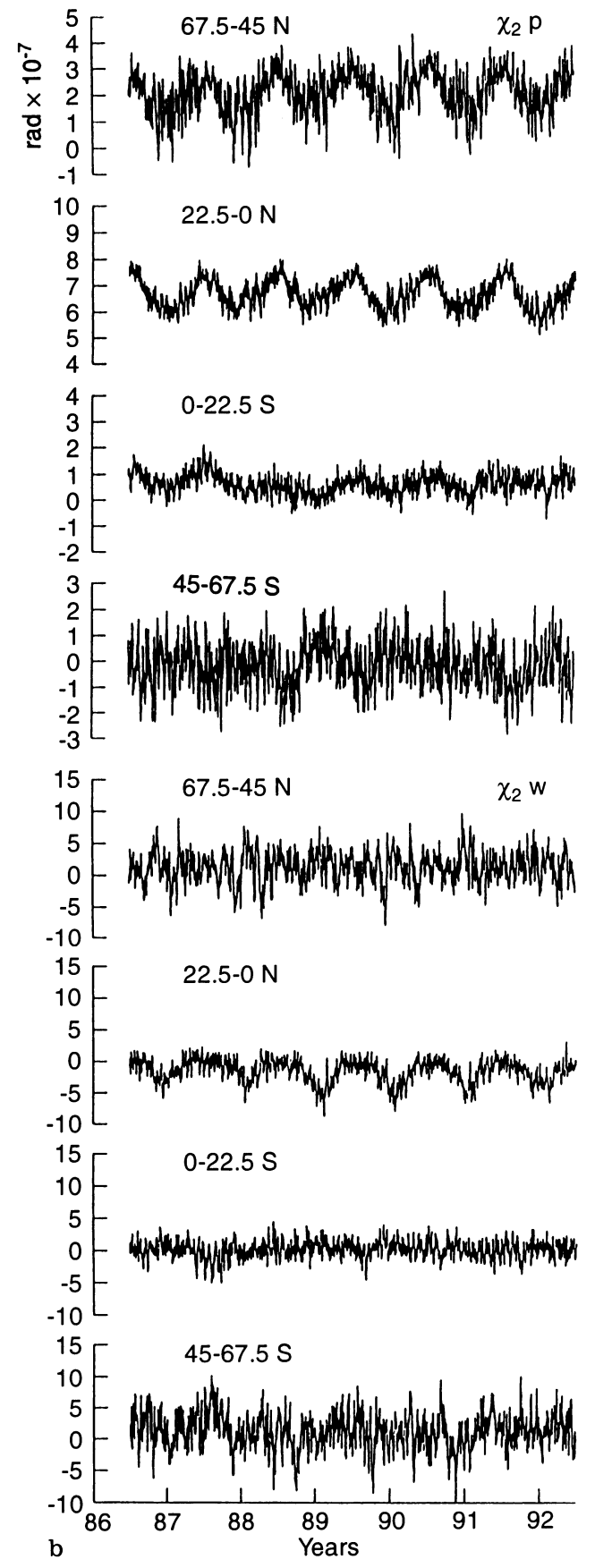

Fig. 1. Examples of variations of the $\mathbf{a}$ pressure and $\mathbf{b}$ wind (b) terms of $\chi_{1}$ and $\chi_{2}$ components of the zonal EAAM (JMA) excitation functions
The square root of these variances is an 'amplitude spectrum' which is proportional to the mean amplitude of a complex-valued oscillation (Kosek, 1995). An oscillation computed using the FT BPF from a complex-valued time series $\chi$ is called prograde for $T>2 \Delta t$ and retrograde for $T<-2 \Delta t$, where $\Delta t$ is the data sampling interval. Prograde and retrograde oscillations in the computed spectra correspond to counterclockwise and clockwise polar motion, respectively.

In Fig. 3 the computed latitude-variable FT BPF amplitude spectra of the complex-valued vector $\chi$ are presented. Distinct latitude dependence of their amplitudes are clearly visible, with maxima mainly in midlatitudes. This result is due to the fact that the centers of maximum variability in surface pressure are located in mid-latitudes, which are attributed the highest weight in the calculation of $\chi$ (Salstein and Rosen, 1989). Latitude-variable amplitude spectra of the wind terms $\chi^{w}$ are several times greater than those of the pressure terms $\chi^{\text {pnib }}$ in their prograde and retrograde parts (Fig. 3). In the case of the whole hemispheres, where the latitudevariable amplitude spectra of the pressure terms are much stronger than those of the wind terms, the situation is the opposite (Kosek et al., 1995; Nastula 1992, 1995a).

Generally, the prograde and retrograde parts of latitude-variable amplitude spectra of the zonal $\chi$ in the considered period ranges have a similar power. Both 

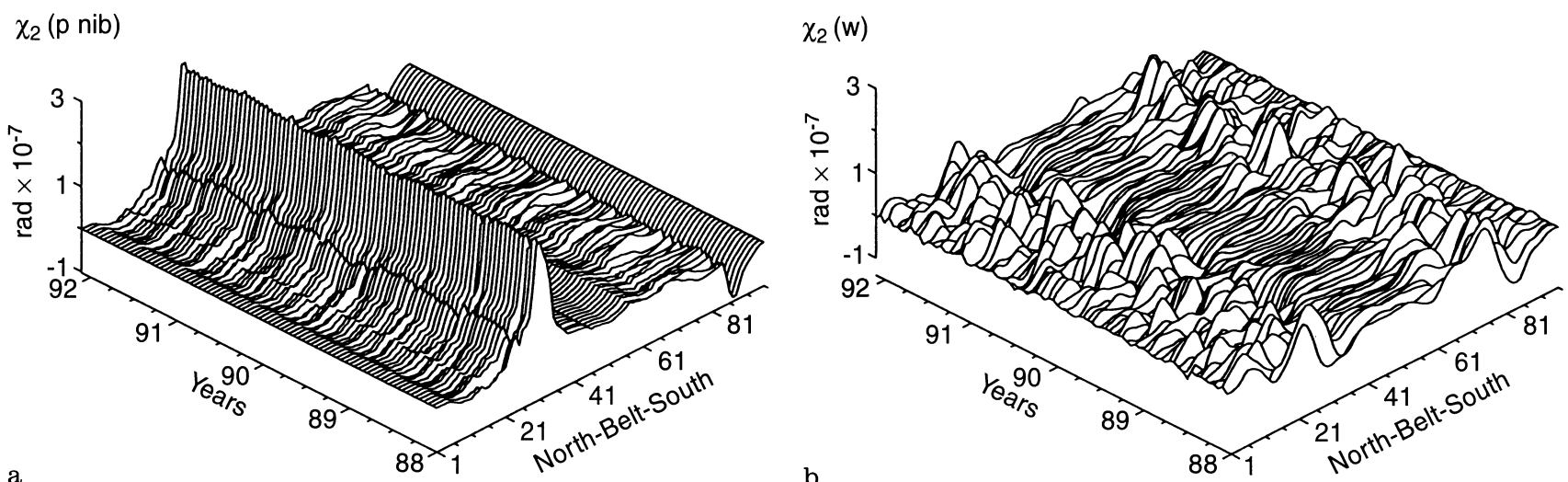

Fig. 2 a, b. Examples of variations of the pressure and wind terms of the $\chi_{2}$ component of the zonal EAAM (JMA) excitation functions in 96 latitude belts of about 2 . width each; a pressure terms, $\mathbf{b}$ wind terms
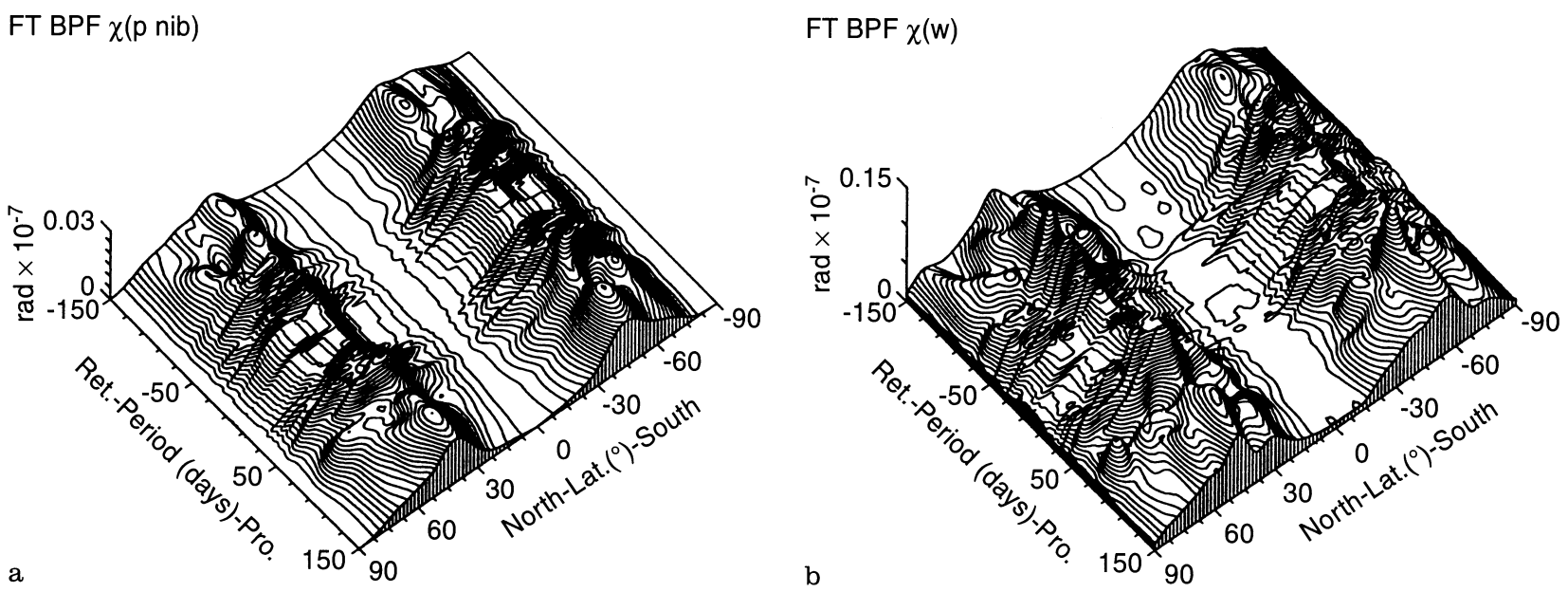

Fig. 3 a, b. Latitude-variable FT BPF amplitude spectrum of the complex-valued a pressure and $\mathbf{b}$ wind terms of the $\chi$ component of the zonal EAAM (JMA) excitation functions; + denotes the prograde oscillations, - denotes the retrograde oscillations

prograde and retrograde parts of these spectra are a little greater in the Southern than in the Northern Hemisphere. The maxima of the amplitude spectra of the pressure terms in the retrograde and prograde parts occur for 40-60 day and 110-120 day oscillations in the latitude belts of $40^{\circ} \mathrm{N}-50^{\circ} \mathrm{N}$ and $40^{\circ} \mathrm{S}-60^{\circ} \mathrm{S}$ (Fig. $3 \mathrm{a}$ ). When the inverted barometric correction in the pressure terms are added then the latitude- variable amplitude spectra decrease, especially in the Southern Hemisphere.

In the considered spectral range the spectra of the wind terms amplitude are more variable than the pressure term spectra mentioned already (Fig. 3b). These spectra show prograde and retrograde oscillations with the periods of about 40-60 days and 80-110 days and the amplitude maxima near $40^{\circ} \mathrm{S}-50^{\circ} \mathrm{S}$ and $40^{\circ} \mathrm{N}-50^{\circ} \mathrm{N}$.

\subsection{Time-variable spectra}

In addition to latitude-variable amplitude spectra, the time variabilities of short period oscillations of the $\chi$ component of the zonal EAAM excitation functions with periods shorter than 150 days were analyzed. The computations of time variable amplitude spectra were made for 4 zones selected out of 8 i.e., for two northern $\left(22^{\circ} .5 \mathrm{~N}-45^{\circ} \mathrm{N}, 45^{\circ} \mathrm{N}-67.5^{\circ} \mathrm{N}\right)$ and two southern $\left(45^{\circ} \mathrm{S}-67.5^{\circ} \mathrm{S} ; 22.5^{\circ} \mathrm{S}-45^{\circ} \mathrm{S}\right)$ belts. These zones were chosen because the latitude-variable amplitude spectra have maximum values in these regions. Most of the northern zones are land areas, covering Europe, Asia with part of Siberia, North America and part of northern Atlantic and Pacific. The southern belts are dominated by mid-latitude southern Pacific.

The method of the FT BPF spectrum was applied again as for the latitude-variable spectra. In this case the time-variable spectrum estimation is given by the following formula (Kosek, 1995):

$$
\hat{S}(t, \phi, T)=\frac{1}{m(T)} \sum_{k=-m(T) / 2}^{m(T) / 2}\left|u_{\phi, t}(T)\right|^{2},
$$

where $u_{\phi, t}(T)$ is defined as in Eq. (1) and $m(T)=j T / \Delta t$ is the number of data within a time interval $j T / \Delta t$ for which $j=2$ was adopted (Kosek, 1995). Setting $j=2$ gives the best time resolution of the time-variable 
spectrum. An increase of $j$ diminishes the time resolution but increases the spectrum accuracy.

Examples of time-variable amplitude spectra of the pressure and wind terms of $\chi$ for the mid-latitude belts, which give the most important contribution to the EAAM excitation functions, are presented in Fig. 4.

In these time-variable amplitude spectra of the pressure terms of $\chi$ for the Northern Hemisphere there are distinct prograde and retrograde oscillations with periods of about 40-80 days and 30-60 days, respectively (Fig. 4a). The amplitude spectra for the Southern Hemisphere are smaller (Fig. 4b).

These oscillations have a distinct annual modulation in mid-latitude belts $22^{\circ} .5 \mathrm{~N}-67^{\circ} .5 \mathrm{~N}$, visible in Fig. 4 , for the latitude belts of $45^{\circ} \mathrm{N}-67^{\circ} .5 \mathrm{~N}$. There are similar oscillations in the amplitude spectra of the pressure with inverted barometric correction terms but they have amplitudes several times smaller in the northern belts and are almost negligible in the southern ones.

In the time-variable amplitude spectra of the northern wind terms we can observe 30-70 day prograde and 3070 day retrograde oscillations (Fig. $4 \mathrm{c}, \mathrm{d}$ ). The strong prograde oscillation with a period of about 40-70 days dominates in the southern wind terms of time-variable amplitude spectra, although the peaks are spread out to 100 day period. Annual variations of these oscillations are visible. In the considered spectrum range the timevariable amplitude spectra of the wind terms have higher amplitudes than those of the pressure terms, similarly to the latitude-variable amplitude spectra discussed already.

\section{Correlation and cross-spectra analysis}

\subsection{Correlation coefficient analysis}

In order to find a quantitative estimation of interactions between the zonal EAAM and geodetic excitation functions, correlation coefficients between them were computed. The geodetic polar motion excitation functions were computed using the modified Wilson and Haubrich time-domain deconvolution formula (Wilson and Haubrich, 1976) from the CSR(93L01) pole coordinate data (Center for Space Research-CSR). In
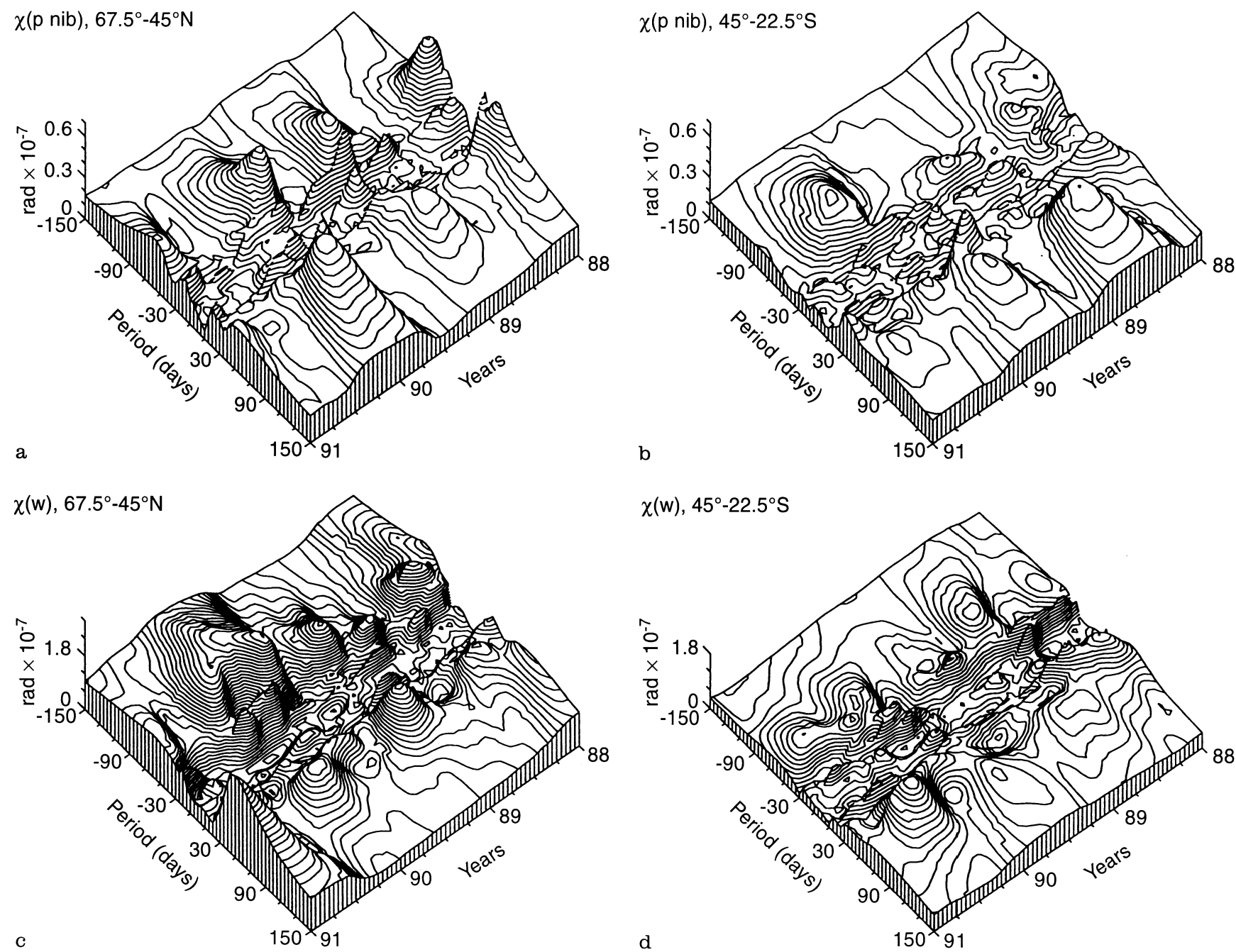

Fig. 4 a-d. Time-variable FT BPF amplitude spectra of the complex-valued $\mathbf{a}$, $\mathbf{b}$ pressure and $\mathbf{c}, \mathbf{d}$ wind terms of the $\chi$ component of the zonal EAAM (JMA) excitation functions; + denotes the prograde oscillations, - denotes the retrograde oscillations 
this computation we adopted a Chandler period of 435 days and a damping coefficient $Q$ of 100 . To remove long period oscillations the computed geodetic polar motion excitation functions were filtered using the Butterworth HPF with a 150-day cutoff period.

Correlation coefficients were computed over a quarter of a year interval, shifted by one eighth of a year through the whole time span of the data. There are about 30 independent points, per one interval and the correlation coefficient of 0.32 is significant at the $95 \%$ confidence level according to the Student's table. The time-variable correlation coefficients between the equatorial components of the geodetic and the zonal EAAM excitation functions were computed for the pressure, pressure with inverted barometric correction, and wind terms. These correlation coefficients vary with latitude and time. Some examples of variations of correlation coefficients between the pressure or wind terms of $\chi_{2}$ components of the zonal EAAM and geodetic polar motion excitation functions are presented in Fig. 5.

The highest positive correlation occurs in the case of the pressure terms, either modified or not, by the inverted barometric correction. These correlation coefficients reach maximum values of $0.5-0.72$ for $\chi_{2}$ and $0.5-0.71$ for $\chi_{1}$. The absolute values of these correlation coefficients for the negative extremes are generally smaller than for the positive ones, and they very rarely exceed 0.5 for both $\chi_{1}$ and $\chi_{2}$ components. The values of correlation coefficient for the wind terms have a random character and the variations of the regional wind terms do not seem to be important for the excitation of polar motion.

Three maxima of these correlation coefficients, for the pressure terms modified or not by inverted barometric correction, occurred during the winters of 1989 , 1990, and 1991. They took place in the latitude zones from $65^{\circ} \mathrm{N}$ to $40^{\circ} \mathrm{N}$ (Fig. 5a). The extreme values of these correlation coefficients occur in the latitude between $40^{\circ} \mathrm{N}$ and $60^{\circ} \mathrm{N}$ with the maxima of $0.5-0.7$ around $50^{\circ} \mathrm{N}-60^{\circ} \mathrm{N}$. For the pressure terms meaningful correlation occurs in the Northern Hemisphere (Fig. 5a). The contribution to the correlation coefficient from the Southern Hemisphere is much smaller; this correlation coefficient very seldom exceeds 0.5 for both $\chi_{1}$ and $\chi_{2}$ components.

As was already shown, the EAAM excitation functions, being the sum of the pressure terms with the inverted barometric correction and the wind terms, is mostly correlated with the geodetic polar motion excitation function (Nastula 1995a, Kosek et al., 1995). Unfortunately, analysis of such a sum is possible only in the case of hemispheric or global variations of EAAM excitation functions. In the case of zonal belts the wind terms dominate in such a sum and its analysis becomes in effect an analysis of the wind terms.

\subsection{Cross-spectra analysis}

A correlation analysis does not give any information about the dependence of the correlation coefficient on frequency. It can be only revealed by cross-spectra. For this analysis the method of the FTBPF cross-spectrum of complex-valued time series was applied (Kosek, 1995). Here the cross-spectrum estimation between two complex-valued time series $\chi^{G}$ and $\chi^{p n i b}$ or $\chi^{w}$ is given by the following formula:

$\hat{S}_{\chi}(t, \phi, T)=\frac{1}{N-2 N_{f}} \sum_{t=N_{f}+1}^{N-N_{f}}\left|u_{\phi, t}(T) \bar{v}_{\phi, t}(T)\right|$,

where $u_{\phi, t}(T)$ is the output computed using the FT BPF from the complex-valued zero-mean $\chi^{\text {pnib }}$ or $\chi^{w}$ time series, $\bar{v}_{\phi, t}(T)$ is the conjugate of the output computed using the FT BPF from the complex-valued zero-mean $\chi^{G}$ time series, $N$ is the number of data points, $N_{f}=10$ is the adopted number of data to be cut off at the ends of the time series, and $T$ is an oscillation period.

Examples of square roots of cross-spectra for the pressure and the wind terms are presented in Fig. 6. These results confirm those obtained from the analysis of correlation coefficients. Maxima of the common energy of polar motion and zonal EAAM excitation functions occur in mid-latitudes for both prograde and

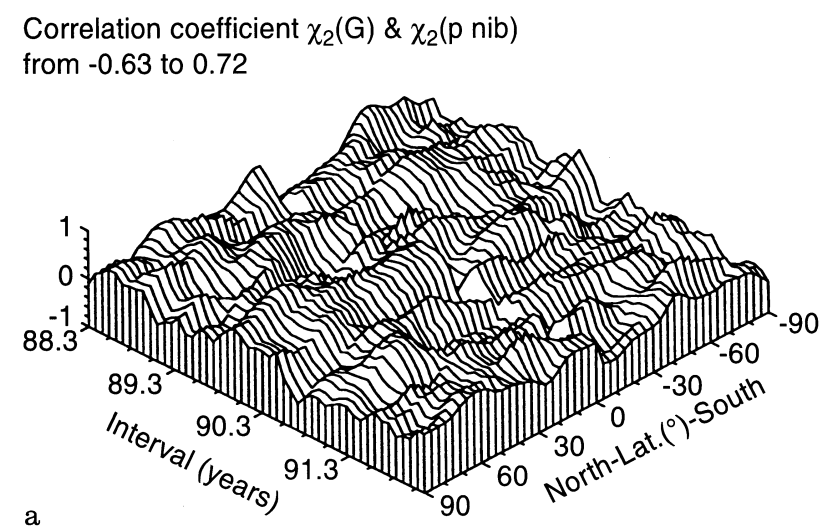

Fig. 5 a, b. Time-variable correlation coefficients between the $\chi_{2}$ components of the zonal EAAM (JMA) and geodetic (CSR93L01) excitation functions, computed for intervals of a quarter of a year, in

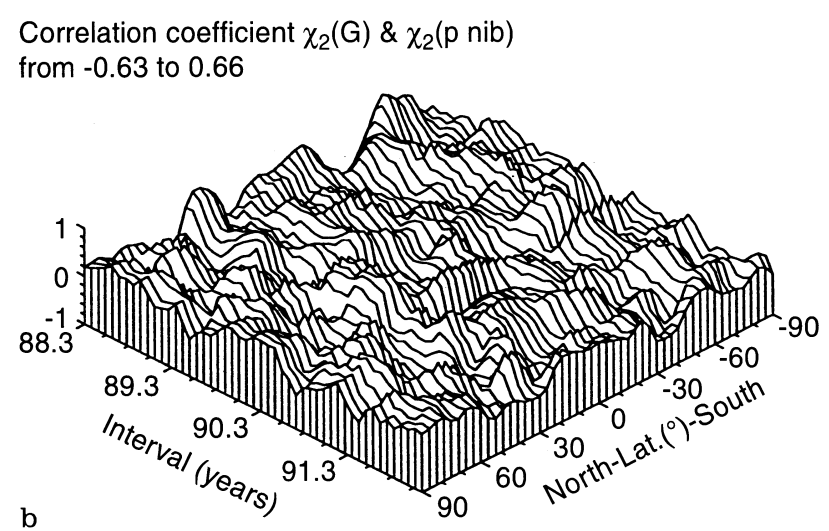

96 latitude belts. a Pressure terms and b wind terms have been taken into account in the zonal EAAM (JMA) excitation functions 


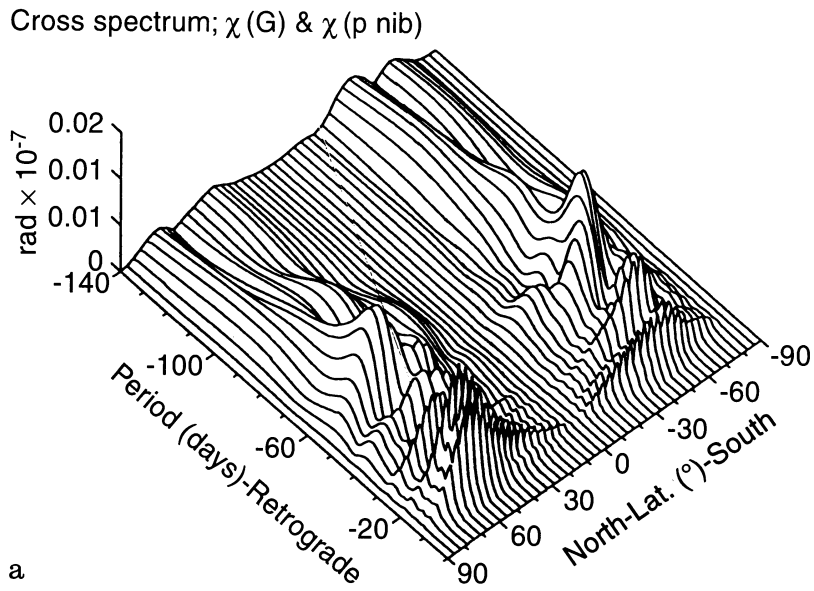

Cross spectrum; $\chi(G) \& \chi(w)$

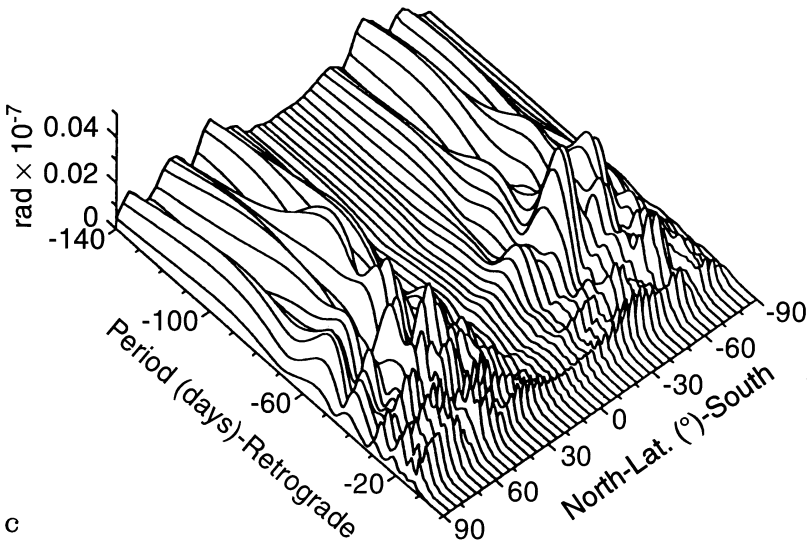

Fig. 6 a-d. Square roots of latitude-variable cross-spectra between the $\chi_{2}$ components of the zonal EAAM (JMA) and geodetic (CSR93L01) excitation functions, computed for intervals of a quarter

retrograde parts of the cross-spectra. The inverted barometric correction of these pressure terms decreases the amplitudes of common oscillations in northern belts and eliminates them in the southern ones. The crossspectra between the $\chi^{w}$ wind terms of the zonal EAAM and the $\chi^{G}$ polar motion excitation functions have a random character. Thus, these $\chi^{w}$ terms do not have any meaningful influence on the variations of polar motion, although some maxima are visible in mid-latitudes.

Large peaks of cross-spectra between the $\chi^{\text {pnib }}$ pressure term and the $\chi^{G}$ polar motion excitation function occur around periods of 20,30,40-50 and 60 days and $80-150$ days in the prograde part. In the retrograde part they reach maximum values around $20,30,40$ and 50-70 days (Fig. 6a,b). The cross-spectra between the $\chi^{w}$ and the $\chi^{G}$ show mainly oscillations with periods of about 40-60 days and 90-110 days in the prograde and of about 20, 30, 40-50, 60-70 and 90 days in the retrograde parts (Fig. 6c,d).

\section{Conclusions}

1. The $\chi_{1}$ and $\chi_{2}$ components of the zonal EAAM (JMA) excitation functions in latitude belts of $22.5^{\circ}$ and

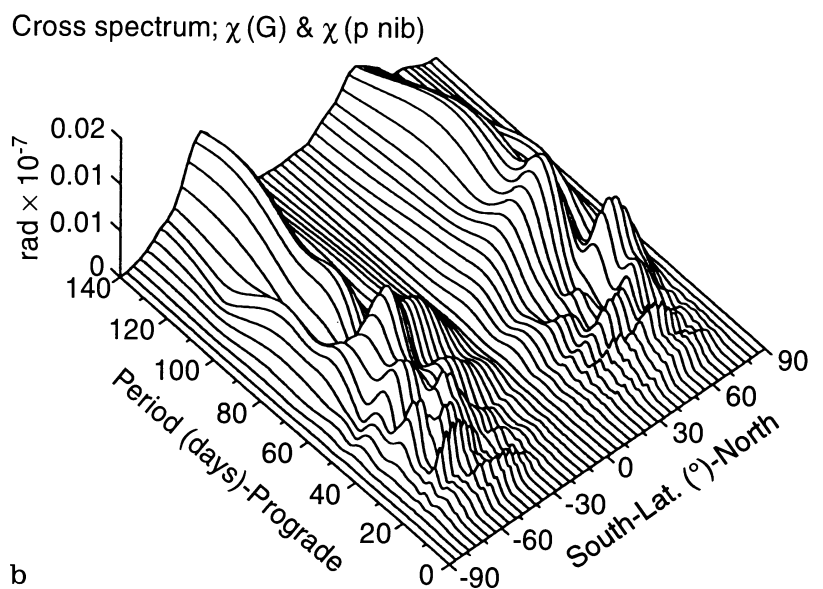

Cross spectrum; $\chi(G) \& \chi(w)$

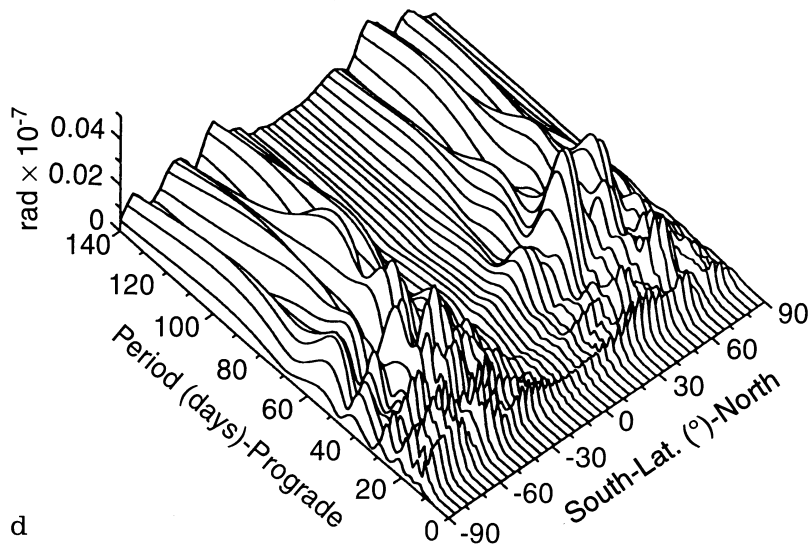

of a year, in 96 latitude belts. The $\mathbf{a}, \mathbf{b}$ pressure, and $\mathbf{c}, \mathbf{d}$ wind terms have been taken into account in the zonal EAAM (JMA) excitation functions

about $2^{\circ}$ latitude widths covering the whole globe were computed for the first time. A large positive maximum in pressure terms of the $\chi_{2}$ components of the zonal EAAM (JMA) excitation functions in the Northern Hemisphere was noticed.

2. The latitude- and time-variable amplitude spectra of the equatorial components $\chi=\left(\chi_{1}+i \chi_{2}\right)$ of the zonal EAAM excitation functions were computed. They are indicative of the character of their variations. The amplitudes of detected oscillations vary in time and latitude and attain maximum values in mid-latitude belts. Generally, the prograde and retrograde parts of latitude-variable amplitude spectra of the zonal EAAM excitation functions have similar amplitudes. The Northern Hemisphere spectra are much stronger than those in the Southern Hemisphere only in the case of the pressure terms with the inverted barometric correction. The 40-70 day prograde oscillations and 30-60 day retrograde oscillations can be observed in time-variable amplitude spectra of the $\chi^{\text {pnib }}$ pressure terms. These oscillations have distinct annual modulation, especially in mid-latitude belts of $22.5^{\circ} \mathrm{N}-67.5^{\circ} \mathrm{N}$.

3. The correlation coefficients between the polar motion and atmospheric excitation functions vary with lati- 
tude and time. The meaningful correlation coefficients between the pressure terms of the zonal EAAM and geodetic excitation functions occur only for the Northern Hemisphere. Their maxima attain the values: -0.6 and from 0.5 to 0.7 .

4. In the cross-spectra between the polar motion excitation functions and pressure terms of the zonal EAAM excitation functions there are peaks of common prograde oscillations with periods around $20,30,40-50,60$ and $80-150$ days and retrograde oscillations with periods around 20,30, 40 and 50-70 days. The cross-spectra between the polar motion excitation functions and the wind terms of the zonal EAAM excitation functions show the prograde oscillations with periods of about 40-60 days and 90-110 days and the retrograde oscillations with periods of about 20, 30, 40, 60-70 and 90 days.

The northern and southern mid-latitude belts are the regions contributing mostly to the short period oscillations of $\chi_{1}, \chi_{2}$ of EAAM excitation functions in the considered period range, for pressure without inverted barometric correction. In most cases the northern belts are land areas, covering Europe, Asia with part of Siberia, North America and part of northern Atlantic and Pacific. The southern belts are dominated by midlatitude southern Pacific. Adding the inverted barometric correction increases the contributions of the EAAM excitation functions for the mid-latitude lands of the Northern Hemisphere. The maximum contribution of the pressure terms of the EAAM excitation function to the polar motion excitation function occur also in the northern mid-latitudes.

Acknowledgements. The support of the Polish State Committee for Scientific Research (Projects 2 P03C 00309 and 2215093 03p/13), the grant from the US-Poland Maria Sklodowska Curie Joint Fund II (Project PAN/SMI-92/97), the Inoue Foundation for Science, and the Japan Society for the Promotion of Science are all gratefully acknowledged. The authors thank Dr. W. Popiński for some subroutines to the program for computation of the complexvalued time series spectra, and Dr. I. Naito and Dr. S. Manabe for the computation program of the hemispheric and zonal EAAM excitation functions. The helpful comments from the reviewers of the manuscript are highly appreciated. The Editor in Chief thanks two referees for their help in evaluating this paper.

\section{References}

Barnes, R. T., R. Hide, A. A. White, and C. A. Wilson, Atmospheric angular momentum fluctuations length of day changes and polar motion, Proc. R. Soc. London, A, 387, 31-73, 1983.
Brzeziński, A., Statistical investigations on atmospheric angular momentum functions and their effects on polar motion, Manuscr. Geodet., 12, 268-281, 1987.

Chao, B. F., Excitation of Earth's polar motion by atmospheric angular momentum variations 1980-1990, Geophys. Res. Lett., 20, (2), 253-256, 1993.

Eubanks, T. M., J. A. Steppe, J. O. Dickey, R. D. Rosen, and D. A. Salstein, Causes of rapid motions of the Earth's pole, Nature, 334, 115-119, 1988.

Hide., R, and J. O. Dickey, Earth's variable rotation, Science., 253, 629, 1991.

Kosek, W., Time variable band filter spectrum of real and complexvalued polar motion series, in Artificial Satellites, Planetary Geodesy, vol 30, 1, The Space Research Center, PAS, Warsaw, Poland, 27-43, 1995.

Kosek, W., J. Nastula, and B. Kolaczek, Variability of polar motion oscillations with periods from 20 to 150 days in 1979-1991, Bull. Géodés. 69, 308-319, 1995.

Kuehne, J., S. Johnson, and C. R. Wilson, Atmospheric excitation of nonseasonal polar motion, J. Geophys. Res., 98, 1997319978, 1993.

Munk, W. H., and G. J. F. McDonald, The rotation of the Earth, a geological discussion, Cambridge University Press, Cambridge, UK.

Naito, I., K. Kikuchi, and K. Yokoyama, Results of estimating the effective atmospheric angular momentum functions based on the JMA global analysis data, Publ. Int. Latit. Obs. Mizusawa, 20, 1-11, 1987.

Nastula, J., Short periodic variations in the Earth's rotation in the period 1984-1990, Ann, Geophysicae, 10, 441-448, 1992.

Nastula, J., Short periodic variations of hemispheric atmospheric angular momentum excitation function in the period 19841992, Ann. Geophysicae, 13, 217-225, 1995a.

Nastula, J., Zonal atmospheric EAAM functions; latitude variations of EAAM difference for 00 and 12 UTC, Reports of Space Research Centre, Report 18, Warsaw, Poland, 1995b.

Nastula, J., D. Gambis, and M. Feissel, Correlated high frequency variations in polar motion and of the length of the day in early 1988, Ann. Geophysicae, 8, 565-570, 1990.

Otnes, R. K., and L. Enochson, Digital time series analysis, Wiley, New York, 1972.

Popiński W., and W. Kosek, The Fourier transform band pass filter and its application for polar motion, Artificial Satellites, Planetary Geodesy, vol 30, 1, The Space Research Centre, PAS, Warsaw, Poland, 9-25, 1995.

Salstein, D. A., and R. D. Rosen, Regional contributions to the atmospheric excitation of rapid polar motions, J. Geophys. Res., 94, 9971-9978, 1989.

Salstein, D. and D. M. Kann, Sub-bureau for atmospheric angular momentum., IERS Annual Report for 1992, III9-III, 1993.

Sunkel, H., and W. Wieser, The global digital terrain model TUG87, Department of Mathematical Geodesy at the Technical University of Graz, Austria, 1987.

Wilson, C. R., and R. A. Haubrich, 1976, Meteorological excitation for the Earth's wobble, Geophys. J.R. Ast. Soc., 46, 707-743, 1976. 